\title{
Icing control model and algorithm for wasteheat exchangers of ventilation systems
}

\author{
Andrew Arbatskiy ${ }^{1, *}$, Andrew Garyaev ${ }^{1}$, and Vasiliy Glasov ${ }^{1}$ \\ ${ }^{1}$ Moscow Power Engineering Institute, 111250 Moscow, Russia
}

\begin{abstract}
Currently, ice control on various heat exchangers to be used for waste heat recovery of discharge air is a rather topical subject because ice building is a factor to reduce efficiency of heat recovery. In such systems, icing always takes place on the side of humid discharge air, with a background of heat exchange between inlet air at temperatures of $-30^{\circ} \mathrm{C}$ and below, and discharge air. To effectively prevent this, it is required to find solutions to problems as follows: study a mechanism of ice building on heat exchange surfaces when interacting with humid air; determine engineering methods to prevent icing, for each type of heat-exchange apparatus, and check efficiency of their operation; develop a mathematical model of ice growth on heat exchange surfaces to enable to vary key parameters (both geometrical and algorithmical ones), determined beforehand, and engineering means aimed at icing prevention.
\end{abstract}

\section{Introduction}

These issues were raised in the paper [1]. Within the framework of this paper, one:

- developed a generalized mathematical model to control icing on a heat exchanger on the warm side of a heat recovery system with an intermediate heat carrier;

- proposed an engineering concept to regulate mode parameters of a heat exchanger to provide for its maximum efficiency at any point of time;

- determined, at a first approximation, an algorithm of improvement of intermediate heat carrier parameters, at various parameters of heating and heated media.

\section{Generalized mathematical model to control icing on a heat exchanger}

A rationale for development of such a mathematical model for operating impact upon icing on heat exchangers within a heat recovery system with intermediate heat carrier (Fig.1) is presented in the paper [1]:

\footnotetext{
* Corresponding author: arbatsky1985@mail.ru
} 


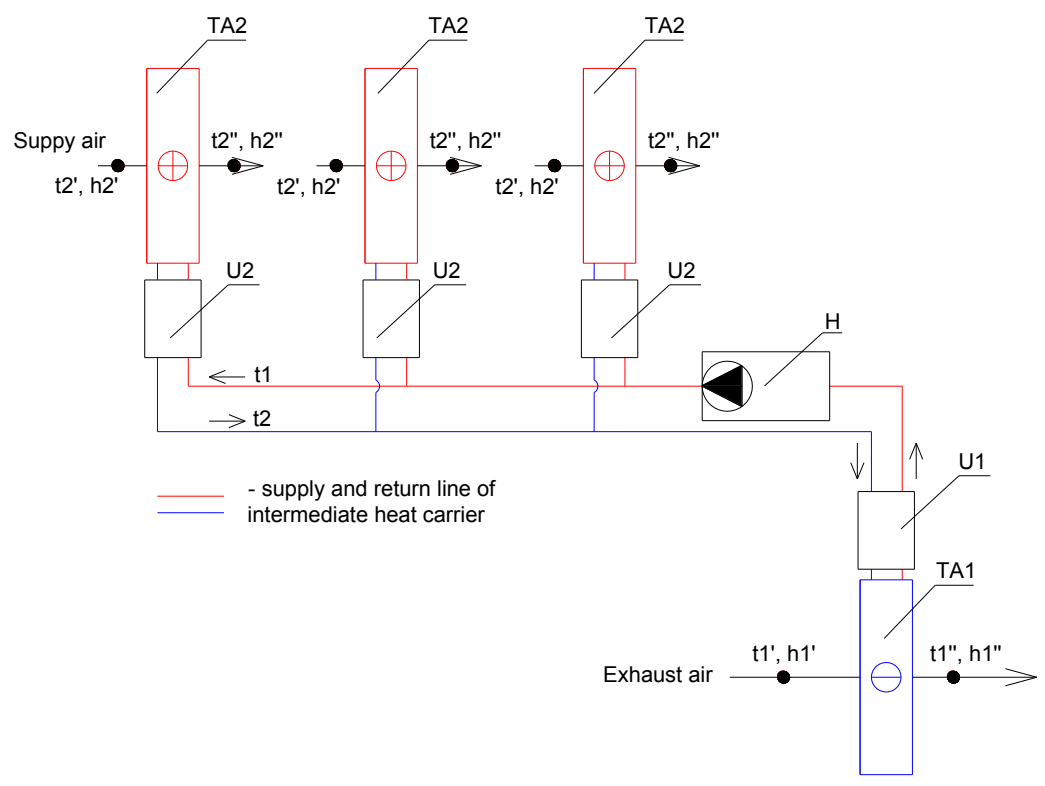

Fig. 1. Principal diagram of a heat recovery system with intermediate heat carrier: TA1 - wasteheat exchanger on the heated side; TA2 - wasteheat exchanger on the heating side; U1 - TA1 regulating unit; U2 - TA2 regulating unit; N - pump group; t2', t2' - in- and outlet temperatures of heat carrier heated; h2', h2'" - in- and outlet total heat of heat carrier heated; t1, t2 - intermediate heat carrier supply and return line temperatures; $\mathrm{t} 1$ ', $\mathrm{t} 1$ ' - in- and outlet temperatures of the heat carrier heating; h1', h1" - in- and outlet total heat of the heat carrier heating.

This type of waste heat exchangers is notable for the lowest thermal effectiveness among other methods to save energy ressources in ventilation systems. This is related to factors as follows:

1) significant losses during heat transmission by means of intermediate heat carrier (IHC);

2) an IHC temperature chart can be improved by equalizing inline parameters of heat exchangers, both on the inlet and discharge side. This, in most cases, results in negative temperatures of IHC, and, consequently, occasional icing on the heat exchanger on the discharge side, especially at higher temperatures of discharge air;

3) Efficiency of the system as a whole depends upon efficiency of an air heater (both inlet and discharge sides). At that, condensate formation in the heat exchanger (discharge side) contributes to, on the one hand, higher efficiency, and on the other hand, when subject to icing, the time-averaged heat exchanger efficiency, on the inlet side, remains unchanged. However, heat current transferred to air by the heat carrier on the inlet side, significantly changes because of changes to temperature head resulting from changes to the temperature chart of the heat carrier.

Before we proceed to creation of a mathematical model to represent ice growth on the TA1 heat exchanger, we have to develop a generalized mathematical algorithm to regulate currents of heat carrier via this apparatus by means of the U1 regulating unit.

Figure 2 shows a principal diagram of the U1 regulating unit: 


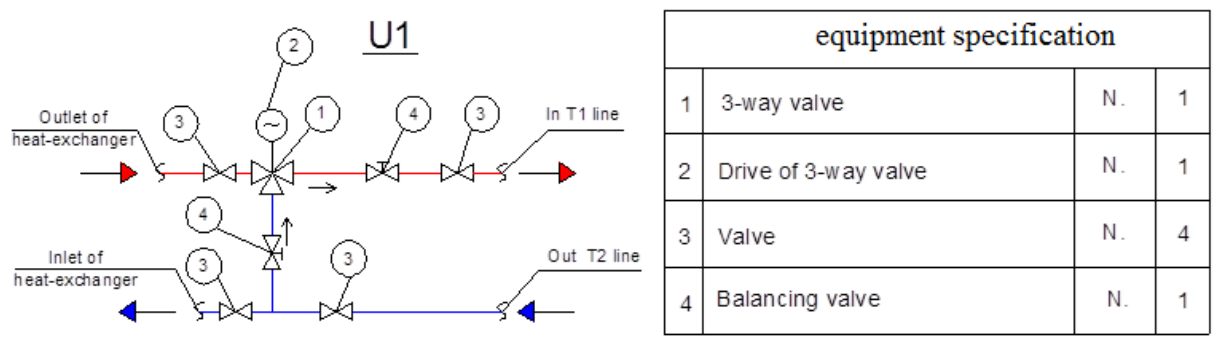

Fig. 2. Principal diagram of the U1 regulating unit.

The diagram enables to control parameters $\mathrm{t} 1$ and $\mathrm{t} 2$, as well as control release of cold IHC into TA1 heat exchanger for removal of external ice. Regulation accuracy is provided due to settings of the balancing valve -4 , because resistance of a bypass where this valve is installed must be considerably higher than that of the heat exchanger to ensure accurate measurement of consumption on the basis of pressure differences on the three-way valve 2. A problem to create an accurate regulating algorithm of this unit is related to generation of the mathematical model of TA 1 heat exchanger icing.

Development of a generalized mathematical model to control U1 is, in the first place, related to qualitative nature of changes to temperature $t_{1}$ during regulations on the three-way valve 2 (Fig. 2). An engineering arrangement of control and regulating mechanisms is presented in Fig. 3.

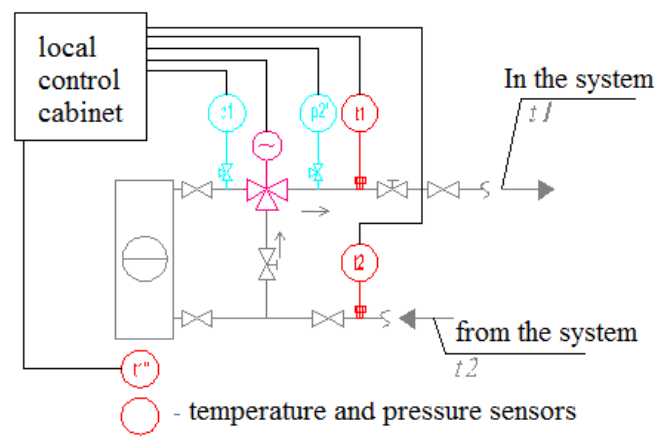

Fig. 3. Principal diagram of the U1 regulating unit.

A position of the three-way valve is determined on the basis of pressure difference:

$$
\Delta P_{m}=\mathrm{p}_{1}-\mathrm{p}_{2}
$$

The regulating scheme of that kind makes it possible to achieve higher accuracy as compared to valve position regulations using an external signal $0-10 \mathrm{~V}$.

Correspondingly, we can write a sequence of the regulating algorithm in the following way:

- When started, all three-way valves are opened; one has to measure pressure difference with the valves opened and write it as an initial constant. Further calculations follow on the basis of this value of $\Delta \mathrm{P}_{\mathrm{m}}$;

- One has to record the following variable at each point of time: $t_{1}, t_{2}, t_{1}$ '”;

- A value of temperature $t_{1}$ at each point of time shall conform to the condition as follows:

$$
t_{1}\left(\mathrm{t}_{2}\right) \geq \mathrm{t}_{2}+\mathrm{k}_{1}
$$

where: $\mathrm{k} 1$ is relationship of heat exchanger specific heat power to a coefficient of thermal 
transmission, ${ }^{\circ} \mathrm{C}$ (actual temperature difference by a liquid provided by an air heater in the design mode).

- Resulting from ice building on the heat exchanger, temperature $t_{1}$ takes a value that does not conform to the condition (2). In this case one begins regulating the three-way valve on the basis of recurrence dependence:

$$
\Delta P_{n}\left(\Delta P_{n-1}\right)=1.013 \Delta P_{n-1}
$$

At that, $\Delta \mathrm{P}_{1}=\Delta \mathrm{P}_{\mathrm{m}}$. The coefficient of 1.013 is related to an averaged empirical characteristic of the three-way valves, diameters of which range from 15 to $50 \mathrm{~mm}$ [2].

- Upon each regulating action of the three-way valve, changes to temperature $\mathrm{t} 1 \mathrm{can}$ be written as follows:

$$
t_{1}(\tau)=\mathrm{k}_{2} \tau^{\varepsilon}
$$

where: $\mathrm{k} 2$ and $\varepsilon$ are constants to be determined experimentally for each type of heat exchangers; $\tau$ is time to be counted from the beginning of each regulating action upon the three-way valve, c;

One has to regulate in such a way so that inequations are always attained:

Table 1. Values of referenced in equations.

\begin{tabular}{|c|c|}
\hline$\lambda$ & $\tau$ \\
\hline$\frac{\mathrm{d} t_{1}(\tau)}{\mathrm{d} \tau}=\left|\mathrm{k}_{2} \varepsilon \tau^{\varepsilon-1}\right| \geq\left|\lambda_{1}\right|$ & $0.1 \tau_{n}$ \\
\hline$\frac{\mathrm{d} t_{1}(\tau)}{\mathrm{d} \tau}=\left|\mathrm{k}_{2} \varepsilon \tau^{\varepsilon-1}\right| \geq\left|\lambda_{2}\right|$ & $\tau_{n} / 2$ \\
\hline$\frac{\mathrm{d} t_{1}(\tau)}{\mathrm{d} \tau}=\left|\mathrm{k}_{2} \varepsilon \tau^{\varepsilon-1}\right| \geq\left|\lambda_{3}\right|$ & $\tau_{n}$ \\
\hline
\end{tabular}

Where: $\lambda_{\mathrm{n}}$ are reference values of a principal derivative for each point of time upon a regulating action.

The principal derivative $t_{1}(\tau)$ in that case characterizes a graph slope angle at each point of time upon regulating actions. With ice on a surface, the rate-of-change of temperature $t 1$ at any movement of the three-way valve will be less than that with no ice, and, consequently, the graph will have a lesser angle of slope;

The algorithm described makes it possible to avoid a need for referencing to specific values of discharge air parameters and practically establish reverence values of the derivative for each type of heat exchangers.

\section{IHC parameters optimization}

To ensure optimal parameters of heat recovery, a temperature chart of IHC must be in the middle between temperature charts of heating and heated media. It means that if a heated medium is air used for ventilation systems, then, IHC parameters can be determined from the equal averaged logarithmical temperature head values on each side, with allowance for efficiency of heat exchangers, due to phase transition to TA1 [3]:

$$
\frac{\varepsilon_{T A 1}}{\varepsilon_{T A 2}} \overline{\Delta t_{T A 1}}=\overline{\Delta t_{T A 2}}
$$


where: $\overline{\Delta t}_{T A 1}$ - averaged logarithmical temperature head for TA1, ${ }^{\circ} \mathrm{C} ; \overline{\Delta t}_{T A 2}$ - averaged logarithmical temperature head for TA2, ${ }^{\circ} \mathrm{C} ; \varepsilon_{T A 1}$ - efficiency of TA1; $\varepsilon_{T A 2}$ - efficiency of TA2.

To solve this problem, in the first place one has to analyze ratio of heat exchangers efficiencies at the same temperature head, but under differing air humidity values. One shall carry out calculations on the basis of averaged values of dry coefficients of transmission, typical for ribbed air heaters at gas velocities making 3-6 m/s k=60 W/m $/ \mathrm{K}$ [4]. This method of calculation will be appropriate, subject to equal flow conditions of heat carriers in TA1 and TA2. In such a way, we can write an equation as follows:

$$
Q_{T A 1}=k F_{1} \overline{\Delta t_{T A 1}}+Q_{\kappa}
$$

where: $\mathrm{k}$ is a dry coefficient of heat transfer (with no humidity taken into account), $\mathrm{W} / \mathrm{m}^{2} / \mathrm{K} ; \mathrm{F}_{1}-$ a heat exchange surface area TA1, $\mathrm{m}^{2} ; \mathrm{Q}_{\mathrm{K}}-$ heat of condensation, $\mathrm{W} ; \mathrm{Q}_{\mathrm{TA} 1}-$ total amount of heat transferred within a heat exchanger per unit time, $\mathrm{W}$.

As $Q_{T A 1}=\Sigma Q_{T A 2}$, subject to equation (6) we obtain as follows:

$$
k F_{1} \overline{\Delta t_{T A 1}}+Q_{\kappa}=k F_{2} \frac{\varepsilon_{T A 1}}{\varepsilon_{T A 2}} \overline{\Delta t_{T A 1}}
$$

where: $F_{2}-$ total surface area of heat exchangers TA2, $\mathrm{m}^{2}$.

From (7) we obtain an equation (8) as follows:

$$
\frac{\varepsilon_{T A 1}}{\varepsilon_{T A 2}}=\frac{F_{1}}{F_{2}}+\frac{Q_{\kappa}}{k F_{2} \overline{\Delta t_{T A 1}}}
$$

It is evident that the second addend on the right is nothing else but ratio of heat of condensation to sensible heat transferred within TA2 that can be written as:

$$
\frac{Q_{\kappa}}{k F_{2} \overline{\Delta t_{T A 1}}}=\frac{\varepsilon_{T A 1} G_{1}\left(\left(h_{1}^{\prime}-h_{1}^{\prime \prime}\right)-c_{p 1}\left(t_{1}^{\prime}-t_{1}^{\prime \prime}\right)\right)}{\varepsilon_{T A 2} G_{2} c_{p 2}\left(t_{2}^{\prime \prime}-t_{2}^{\prime}\right)}
$$

where: $c_{p 1}$ and $c_{p 2}$ are mean specific heat values of air, $J / k g / K ; G_{1}$ and $G_{2}$ represent consumption values of heating and heated heat carriers, $\mathrm{kg} / \mathrm{s}$.

Inserting (9) into (8), we obtain the final equation as follows:

$$
\frac{\varepsilon_{T A 1}}{\varepsilon_{T A 2}}=\frac{F_{1}}{F_{2}}+\frac{\varepsilon_{T A 1}}{\varepsilon_{T A 2}} \frac{G_{1}\left(\left(h_{1}^{\prime}-h_{1}^{\prime \prime}\right)-c_{p 1}\left(t_{1}^{\prime}-t_{1}^{\prime \prime}\right)\right)}{G_{2} c_{p 2}\left(t_{2}^{\prime \prime}-t_{2}^{\prime}\right)}
$$

It may be affirmed that we always will obtain equation of averaged logarithmical temperature heads, subject to equation of averaged values of temperature heads, basing upon which we can write a ratio as follows:

$$
\frac{F_{1}}{F_{2}}+\frac{t_{1}-t_{2}^{\prime \prime}+t_{2}-t_{2}^{\prime}}{t_{1}^{\prime \prime}-t_{2}+t_{1}^{\prime}-t_{1}} \frac{G_{1}\left(\left(h_{1}^{\prime}-h_{1}^{\prime \prime}\right)-c_{p 1}\left(t_{1}^{\prime}-t_{1}^{\prime \prime}\right)\right)}{G_{2} c_{p 2}\left(t_{2}^{\prime \prime}-t_{2}^{\prime}\right)}=\frac{t_{1}-t_{2}^{\prime \prime}+t_{2}-t_{2}^{\prime}}{t_{1}^{\prime \prime}-t_{2}+t_{1}^{\prime}-t_{1}}
$$


From the expression (11), a formula can be derived to calculate an average temperature of IHC:

$$
\frac{t_{1}+t_{2}}{2}=0.5 \frac{\frac{F_{1}}{F_{2}}\left(t_{1}^{\prime \prime}+t_{1}^{\prime}\right)-\frac{G_{1}\left(\left(h_{1}^{\prime}-h_{1}^{\prime \prime}\right)-c_{p 1}\left(t_{1}^{\prime}-t_{1}^{\prime \prime}\right)\right)}{G_{2} c_{p 2}\left(t_{2}^{\prime \prime}-t_{2}^{\prime}\right)}\left(t_{2}^{\prime \prime}-t_{2}^{\prime}\right)+t_{2}^{\prime \prime}+t_{2}^{\prime}}{\frac{F_{1}}{F_{2}}-\frac{G_{1}\left(\left(h_{1}^{\prime}-h_{1}^{\prime \prime}\right)-c_{p 1}\left(t_{1}^{\prime}-t_{1}^{\prime \prime}\right)\right)}{G_{2} c_{p 2}\left(t_{2}^{\prime \prime}-t_{2}^{\prime}\right)}+1}
$$

Using dependence (12) for various schemes of heat recovery, we can always achieve the best IHC temperature values.

\section{References}

1. A. A Arbatskiy, V. S. Glasov, Status and prospects of the development of electro- and heat technology 2, 320 (2017) [in Russian]

2. Esbe. Catalogue of $3 \mathrm{G}$ three-way valves characteristics [in Russian]

3. V. P. Isachenko, V. A. Osipova, A. S. Sukomel, Heat transmissio (Energy, Moscow, 1975) [in Russian]

4. A. M. Baklastov, V. A. Gorbenko, O. L. Danilov, Industrial-scale heat-mass-exchange apparatus and processes (Energoatomizdat, Moscow, 1986) [in Russian] 